\title{
Micromachined Electron Tunneling Infrared Sensors
}

\author{
T.W. Kenny, W.J. Kaiser, J.A. Podosek, H.K. Rockstad, and J.K. Reynolds \\ Center for Space Microelectronics Technology \\ Jet Propulsion Laboratory, California Institute of Technology, Pasadena, CA 91109
}

\section{Introduction}

Many infrared detectors have been developed over the last 40 years. ${ }^{1-3}$ In most cases, they may be classified as either quantum or thermal detectors, depending upon whether the incoming radiation is converted to excitations which are collected, or is converted to heat and detected through changes in temperature. ${ }^{4}$ Quantum detectors must separate optically generated excitations from thermally generated excitations, and therefore require cooling for detection of infrared photons $(\lambda>5 \mu \mathrm{m})$. In contrast, thermal detectors which operate at room temperature can be very sensitive to infrared radiation. Because of restrictions on power consumption or the availability of cryogens, many applications are driven towards the use of uncooled thermal infrared detectors.

The development of thermal infrared detectors is limited by the availability of sensitive, low-mass thermometers. Phase transitions in solid state materials provide the sensitive thermometer for detectors such as pyroelectrics and HiTc bolometers. Other thermal infrared detectors have been based on thermocouple thermometers, or in the case of the Golay Cell, the thermal expansion of a small volume of trapped gas. In general, the thermodynamical optimization of these detectors has been carried out for many years and is nearly complete.

All thermal infrared detectors may be parameterized by the heat capacity of the active element, the thermal conductance between the active element and the surroundings, the response coefficient of the thermometer, and the noise spectra which arise from the anticipated noise sources. Using these parameters, it is straightforward to predict performance of any infrared sensor. It is possible, for example, to calculate the responsivity of a thermal infrared detector from these parameters. The user community is most interested in calculations of the Noise Equivalent Power (NEP), which is used to evaluate the utility of the proposed detector.

New thermal infrared detectors may be developed whenever a new solid-state thermometer, or a new fabrication technology becomes available. Recent examples include the use of silicon micromachining to construct miniature infrared detectors based on thermal isolation of pyroelectric materials, metal-film thermometers, high-temperature superconducting oxide films, and thermocouples. In some cases, these devices offer performance characteristics that approach fundamental limitations to the sensitivity of infrared detectors.

The Golay Cell consisted of a small cavity filled with gas at room temperature. The cavity is separated from the surroundings by a window and a thin, flexible membrane. $5-7$ The membrane was coated on one side with a thin metallic film, which has significant absorption throughout the infrared whenever the sheet resistance of the film is approximately half of the impedance of free space. ${ }^{8-10}$ The trapped gas in the Golay cell was heated by contact with the membrane, and expanded thermally, which forced the membrane to deflect outward. This deflection is usually detected with optical or capacitive displacement transducers. ${ }^{11}$ At present, these detectors are bulky, fragile, difficult to fabricate, and expensive. Nevertheless, they have been widely used, primarily because of their improvement in NEP over all other uncooled detectors in the mid to far infrared. 
The advantage to operation of an infrared detector in this manner may be described as follows. All thermal infrared detectors benefit from reduction in the heat capacity of the thermometer. In the Golay cell, the gas coupled to the membrane displacement represents an easily manufactured, very low heat capacity thermometer. In addition, this thermometer is very sensitive, in that the volume of an ideal gas at room temperature changes by $0.3 \%$ for each $1 \mathrm{~K}$ change in temperature. The low heat capacity and high sensitivity combine to make the Golay cell infrared detector as much as 5-10 times more sensitive than typical pyroelectric detectors.

Attempts to miniaturize the Golay cell for incorporation into focal plane arrays have been unsuccessful because of scaling laws which relate the sensitivity of conventional miniature displacement transducers and their active area. The resulting detectors were interesting, but not as useful as arrays of pyroelectric or bolometric detectors.

\section{Prototype Tunneling Infrared Sensor Theory}

With the above considerations in mind, we have begun the development of an improved Golay cell. This new sensor is constructed entirely from micromachined silicon components. In this device, a silicon oxynitride $\left(\mathrm{SiO}_{\mathrm{x}} \mathrm{N}_{\mathrm{y}}\right)$ membrane is deflected by the thermal expansion of a small volume of trapped gas. To detect the motion of the membrane, we use an electron tunneling displacement transducer. ${ }^{12,13}$ This sensor, like the assemblies used in Scanning Tunneling Microscopy (STM), ${ }^{14,15}$ detects electrons which tunnel through the classically forbidden barrier between a tip and a surface. As in the STM, the electron current is exponentially dependent on the separation between the tip and the surface. The sensitivity of tunneling transducers constructed and operated by our group is typically better than $10^{-3}$ $\AA \sqrt{\mathrm{Hz}}$. Through use of the electron tunneling transducer, the scaling laws which have prevented the miniaturization of the Golay cell are avoided. This detector potentially offers low-cost fabrication, compatibility with silicon readout electronics, and operation without cooling. Most importantly, this detector may offer better sensitivity than any other uncooled infrared sensor, with the exception of the original Golay Cell.

The responsivity and noise of the electron tunneling infrared detector may be calculated as for any thermal detector. It is important to note that this particular prototype was not optimized to achieve the best possible sensitivity.

A cavity of area $(0.1 \mathrm{~cm})^{2}$ and thickness $0.015 \mathrm{~cm}$, filled with air at atmospheric pressure is trapped between a pair of silicon wafers, one of which has been etched through to a $0.5-\mu \mathrm{m}$ thick silicon oxynitride membrane as shown in Fig 1. The outer surface of the membrane is coated with $\sim 70 \AA$ of gold to serve as an electrode for tunneling as well as an efficient absorber of infrared radiation. The heat capacities of the gas, membrane, and gold films sum to $\mathrm{C}=7.9 \times 10^{-7} \mathrm{~J} / \mathrm{K}$, and the thermal conductances between these elements and the surroundings sum to $\mathrm{G}=1.9 \times 10^{-4} \mathrm{~W} / \mathrm{K}$. In this case, the heat capacity is dominated by the membrane, while the thermal conductance is dominated by conduction through the trapped gas to the window. The mechanical properties of the membrane may be calculated, given its dimensions. ${ }^{16}$ The coefficient of motion (change in position per unit change in temperature) of the center of the membrane is approximately $1 \times 10^{-4} \mathrm{~cm} / \mathrm{K}$, assuming that air is an ideal gas at room temperature and pressure.

A tunneling transducer is used to measure the deflection of the membrane. When biased at fixed voltage, the tunneling current has the following dependence ${ }^{17}$ on tip-substrate separation: 


$$
I=I_{0} \exp (-\beta \sqrt{\Phi} s)
$$

where $\Phi$ is the tunnel barrier height in $\mathrm{eV}, \mathrm{s}$ is the tip-membrane separation in $\AA$, and $\beta$ is a conversion factor with a value of $1.025 \mathrm{eV}^{-1 / 2} \AA^{-1}$. The temperature coefficient (fractional change in current per unit change in temperature) of the trapped gas and tunnel sensor is $\alpha=(1 / \mathrm{I})(\partial \mathrm{V} / \partial \mathrm{T})=2.3 \times 10^{4} / \mathrm{K}$, for $\Phi=5$ $\mathrm{eV}$. This large temperature coefficient is a product of the large thermal expansion of gases at room temperature, and the extreme sensitivity of vacuum tunneling to changes in relative position. For comparison, $\alpha$ for a good resistance bolometer is of order unity.

With this information, it is possible to calculate the response of the sensor per unit input power. The responsivity of the electron tunneling infrared detector is given by :

$$
S=\frac{I \alpha}{\left(G^{2}+(\omega C)^{2}\right)^{1 / 2}}=1.2 \times 10^{-1} \mathrm{~A} / \mathrm{W} \quad(\omega=0) \text {. }
$$

It is important to remember that the responsivity is not in itself a useful figure of merit for comparisons between infrared sensors because it contains no information about the noise of the sensor.

We shall consider the contributions of several kinds of noise. For each source, an expression for the square of the Noise Equivalent Power, (NEP) ${ }^{2}$, appears. The NEP is defined as the signal power that would be detected with a unity signal-to-noise ratio in the presence of a given source of noise. The expression for the NEP refers to a bandwidth of $1 \mathrm{~Hz}$. Since the noise sources being considered are broadband, reducing the bandwidth of the measurement (increasing the inlegration time) is expecled to improve the sensitivity. An absorber efficiency of $100 \%$ is assumed in this expression.

The NEP of a thermal infrared decector is given by: 18

$$
(\mathrm{NEP})^{2}=4 \mathrm{k}_{\mathrm{B}} \mathrm{T}^{2} \mathrm{G}+\frac{2 \mathrm{e}\left(\mathrm{G}^{2}+(\omega \mathrm{C})^{2}\right)}{1 \alpha^{2}}+16 A \sigma \mathrm{k}_{\mathrm{B}} \mathrm{T}^{5} \text {. }
$$

The first term arsi, due to temperature fluctuations in the trapped gas, and is common to all thermodynamic wjects which are isolated from a temperature reference by a fixed duemal cunderctance. The sciond wrm is due to shot noise in the tunnel current, and is equivalent to a Johnson noise term which is ciummonly found when analyzing resistance bolometers. The third term is due to statistical fluctuations in the arrival of photons, and is the fundamental limit to the sensitivity of all thermal infrared sensors. In

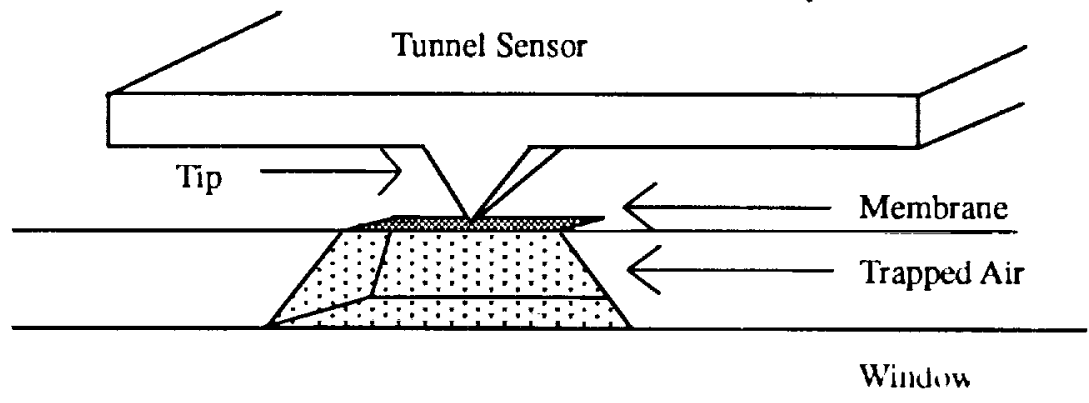

Fig. 1 This drawing shows the design of the prototype tunneling infrared sensor. Infrared radiation, incident from the bottom of the figure, is absorbed in a thin metal film deposited on the membrane and induces thermal expansion in the trapped gas. The deflection of the membrane is monitored by the iunneling transducer. 
this expression, $\mathrm{k}_{\mathrm{B}}=1.38 \times 10^{-23} \mathrm{~W} / \mathrm{K}$ is Boltzmann's constant, $\mathrm{T}$ is the operating temperature, $\omega$ is the modulation frequency, $A$ is the absorbing area of the detector, and $\sigma=5.67 \times 10^{-12} \mathrm{~W} /\left(\mathrm{cm}^{2} \mathrm{~K}^{4}\right)$ is the Stefan-Boltzmann constant.

It is important to note the role played by the large response coefficient, $\alpha$, in this expression. Since $\alpha$ is larger than $10^{4} \mathrm{~K}^{-1}$, the shot noise term in (3) is greatly reduced until $\omega \mathrm{C}$ becomes much larger than $G$.

For the dimensions and operating parameters of this infrared sensor, the NEP associated with thermal noise, shot noise, and photon noise is $3.0 \times 10^{-11} \mathrm{~W} / \sqrt{\mathrm{Hz}}, 1.4 \times 10^{-13} \mathrm{~W} / \sqrt{\mathrm{Hz}}$, and $5.2 \times 10^{-12}$ $\mathrm{W} / \sqrt{\mathrm{Hz}}$, respectively, at $\omega \ll \mathrm{G} / \mathrm{C}$. The thermal noise dominates the NEP for all modulation frequencies below $10 \mathrm{kHz}$.

Noise in amplifiers should not play an important role due to the large transducer sensitivity. Commercial operational amplifiers are readily available that contribute less than shot noise to the NEP.

If we sum these contributions to the noise, and consider the $50 \%$ efficiency of the absorber, the predicted NEP of the prototype is $7 \times 10^{-11} \mathrm{~W} / \sqrt{\mathrm{Hz}}$ at chopping frequencies below $10 \mathrm{kHz}$. At frequencies below $10 \mathrm{kHz}$, the NEP is dominated by thermal fluctuations. At frequencies above $10 \mathrm{kHz}$, the contribution from shot noise dominates the other sources of noise and degrades the NEP of the detector. The calculated contributions to the NEP of the prototype are plotted as a function of frequency in Fig. 2. For comparison, the NEP of a commercially available pyroelectric infrared sensor is shown as well.

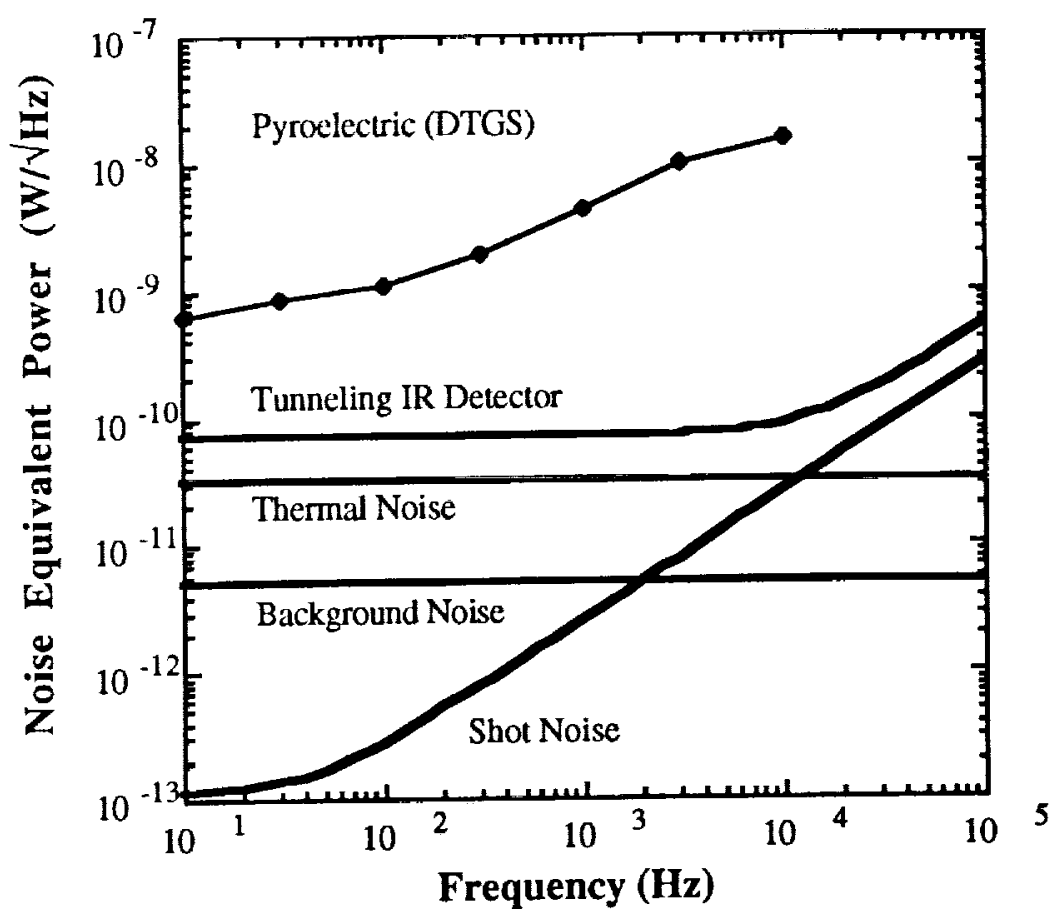

Fig. 2 This graph shows the contributions to the NEP of the tunneling infrared detector. The NEP of a typical pyroelectric infrared detector is shown for comparison 


\section{Prototype Tunneling Infrared Sensor}

We have designed and constructed a prototype electron tunneling infrared sensor based on an early version of our tunneling transducer. Micromachining has been used in this case to produce $\mathrm{cm}$-scale components with $\mu \mathrm{m}$-scale precision. In contrast to conventional tunneling devices, the relative position of the electrodes is controlled through use of electrostatic forces applied between the elements. The electrostatic forces induce deflection of a micromachined silicon cantilever spring. Use of the electrostatic actuator is important because of insensitivity to thermal drifts and immunity to creep. Also the response of the electrostatic actuator is a function only of the geometry and mechanical properties of the device, whereas the response of piezoelectric actuators is also dependent on the characteristics of the material, which may not be reproducible between devices or over time. Finally, the electrostatic actuator may be miniaturized more easily because the scaling laws are known exactly, and the fabrication is less complex than for the piezoelectric actuator. Figure 3 shows a sketch of the micromachined infrared sensor components. The components are approximately $4 \mathrm{~cm}^{2}$ in area. The inner rectangular area of the folded cantilever spring can be deflected upward or downward relative to the outer segments by application of a voltage between the large electrode and a corresponding deflection counter-electrode which is deposited on another component of the sensor. Given the mechanical properties of the silicon as well as the dimensions of the spring structure, we can calculate the properties of the spring. Because the spring constant scales rapidly with the dimensions of the legs of the folded cantilever, its characteristics can easily be tailored to meet the needs of a specific application. Various methods for manufacturing a suitable tunneling tip are available. 8,19 We have formed silicon tips directly from the substrate by undercutting a $60 \mathrm{~mm} \times 60 \mathrm{~mm}$ square of $\mathrm{SiO}_{2}$ with EDP. The active surfaces of all electrodes are prepared by evaporation of $3000 \AA$ thick Au films through a micromachined shadow mask.

Once the device is assembled, a voltage is applied to the electrostatic deflection electrodes. This deflection voltage produces an attractive force between the electrodes, which reduces the electrode separation. When the tip is within several $\AA$ of the membrane, a tunnel current is established. Active regulation of the tip-electrode separation using feedback control of the tunneling current is carried out as

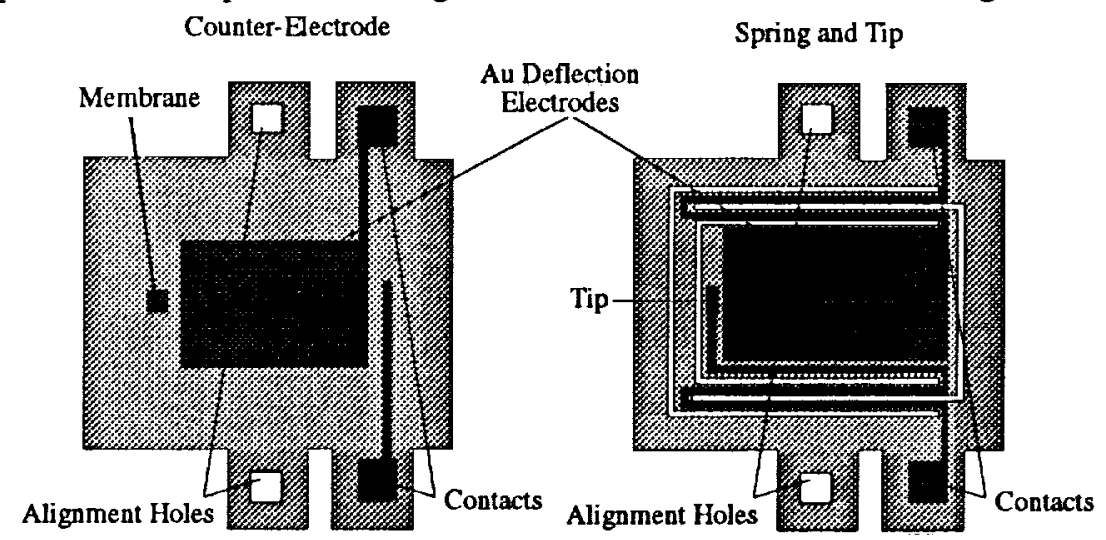

Fig. 3 This drawing shows the design of the micromachined silicon tunneling transducer. A folded cantilever spring, which supports a micromachined tip and a deflection electrode, is suspended over the micromachined counterelectrodes. Electrostatic forces between the deflection electrodes are used by a feedback loop to control the tunneling current. Variations in the deflection voltage occur with the absorption of IR radiation. 
for STM. The absorption of infrared radiation causes thermal expansion of the trapped gas, and a resulting change in the tunneling current. The feedback circuit responds by reducing the deflection voltage, thereby forcing the cantilever to track the motion of the membrane. Infrared signals are monitored by recording the variations in deflection voltage.

The micromachined tunneling transducer was operated and characterized. While operating in the laboratory, displacement sensitivities of $10^{-3} \AA / \sqrt{\mathrm{Hz}}$ at $10 \mathrm{~Hz}$ and $10^{-4} \AA / \sqrt{\mathrm{Hz}}$ at $1 \mathrm{kHz}$ were measured.

The prototype infrared sensor was made operational, and infrared response was observed with a variety of laboratory blackbody sources. A careful calibration was carried out by chopping between $300 \mathrm{~K}$ and $77 \mathrm{~K}$ blackbodies with area $1 \mathrm{~cm}^{2}$ located $10 \mathrm{~cm}$ from the sensor. Considering the geometric factors and allowing for atmospheric transmittance from source to sensor, the average modulated signal power at the sensor was $1.6 \times 10^{-7} \mathrm{~W}$. The signal to noise ratio at the chopping frequency of $10 \mathrm{~Hz}$ and bandwidth of $1 \mathrm{~Hz}$ was measured to be 200, so the experimental NEP of the prototype infrared sensor is $8 \times 10^{-10}$ $\mathrm{W} / \sqrt{\mathrm{Hz}}$. Noise in this prototype was dominated by the sensitivity of the transducer to mechanical vibration.

In the case of the prototype infrared sensor, the resonant frequency of the large cantilever that was used to track the displacement of the membrane was too small. As a result, the separation between the tunneling contacts was not well controlled, and true tunneling only occurred during a small fraction of the operating time. In addition, the transducer was sensitive to vibration, and the vibration signals increased the total noise during measurements.

\section{Improved Tunneling Infrared Sensor}

To construct an improved infrared sensor, we have chosen to dispense with the cantilever altogether, and apply the rebalance force from the feedback circuit to the membrane directly. A drawing of the improved infrared sensor is shown in Fig. 4. The device is operated by applying a deflection voltage to the membrane to pull it to within tunneling distance of the tip. A standard wide-bandwidth STM feedback loop is used to control the deflection voltage so as to maintain the tunneling current at a constant value. Upon the absorption of infrared radiation, the pressure in the trapped gas will change, affecting the force applied to the membrane by the gas. The feedback circuit will respond to this by adjusting the rebalance force so as to keep the membrane in the same position.

We have built and operated an infrared sensor based on this improved design. A preliminary calibration was carried out, giving an NEP of at least 2-4 $\times 10^{-10} \mathrm{~W} / \sqrt{\mathrm{Hz}}$. The absorber used in this particular sensor was not well optimized; further reductions in NEP by as much as a factor of 3 may occur with an optimized absorber. A complete characterization of this sensor is presently under way.

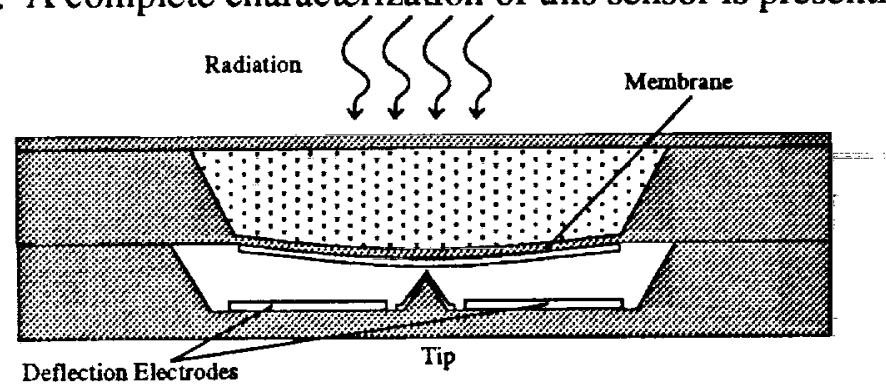

Fig. 4 This drawing shows the design of the improved tunneling infrared sensor. Electrostatic deflection is used by a feedback loop to control the position of a silicon nitride membrane. Variations in this deflection voltage are required to compensate for IR-induced variations in the pressure of the trapped gas. 
The performance of this improved sensor exceeds that of the prototype in several important respects. First, the sensitivity to vibration is reduced because the mass of the moving transducer element is reduced by 5 orders of magnitude and the resonant frequency is increased by 3 orders of magnitude. Second, the bandwidth of the feedback loop that controls the transducer may now be increased by more than two orders of magnitude. Finally, the elimination of the folded cantilever allows the design of a simpler structure; this structure may be more easily fabricated and miniaturized. The improved sensor occupies only as much real estate on the focal plane as the absorber, and may be more easily arrayed.

The measured sensitivity of the tunneling infrared sensor is already competitive with the best commercial pyroelectric sensors. The NEP of this detector may be further improved by reducing G. Techniques for reducing $\mathrm{G}$ include using xenon for the gas, reducing the area of the membrane, and increasing the thickness of the cavity to reduce the surface area-to-volume ratio. These straightforward modifications will allow reductions in the NEP by an additional factor greater than 5 . With these modifications, the first uncooled infrared detector with performance approaching background limits may be realized.

This detector can be made to operate at lower temperatures through the use of a more ideal gas, such as helium. The contribution to the NEP from thermal fluctuations should decrease linearly with temperature. Below $100 \mathrm{~K}$, other technologies, particularly HiTc bolometers, $\mathrm{HgCdTe}$, and $\mathrm{InSb}$, should still be superior to this detector.

The tunneling infrared sensor, being assembled entirely from micromachined silicon, can be miniaturized and batch-fabricated or integrated into arrays. Individual detectors of diameter less than 100 $\mu \mathrm{m}$ can be assembled without development of additional technology by scaling the design of the improved infrared sensor. Each element would require a tunneling transducer with its own feedback circuit; therefore, integration of sensor and control electronics is necessary.

There are many potential applications of this detector since it is sensitive to radiation throughout the infrared. However, it appears ideally suited to applications that benefit from or require the use of an uncooled detector in measurement of radiation in the mid and far infrared, such as NASA planetary missions.

\section{Tunneling Accelerometer}

The tunneling transducer has also been investigated for application to measurement of acceleration. For example, the prototype micromachined tunneling transducer featured acceleration sensitivity of approximately $10^{-7} \mathrm{~g} / \sqrt{\mathrm{Hz}}$ at $10 \mathrm{~Hz}$.

We have begun the development of an improved tunneling accelerometer which is intended to offer sensitivity of $10^{-8} \mathrm{~g} / \sqrt{\mathrm{Hz}}$ at all frequencies between $5 \mathrm{~Hz}$ and $1 \mathrm{kHz}$. This improved accelerometer features a lightweight, wide-bandwidth cantilever that is used to follow the motion of a suspended $1 \mathrm{mg}$ proof mass. In this device, the feedback forces are applied to the cantilever instead of the proof mass. Since the feedback loop does not attempt to control the position of the proof mass, the loop may be operated both below and above the resonance of the proof mass. The bandwidth of the feedback loop is limited by the resonance of the cantilever, which is expected to exceed $10 \mathrm{kHz}$.

As for the infrared detector, it is important to consider not only the responsivity, but also the noise associated with this accelerometer. The performance of this miniature accelerometer is limited by thermal noise-induced vibrations in both the proof mass and the cantilever. We have carried out a thorough 
analysis of the contributions of thermal noise to the sensitivity of the tunneling accelerometer. By separating the functions of cantilever and proof mass, the physical parameters of these components may be independently optimized to reduce thermal noise while preserving acceleration sensitivity. The details of this analysis are planned to be published elsewhere.

We thank J. Maserjian, and P.J. Grunthaner for their encouragement and support. We are indebted to K. Potter and D. Rutledge for assistance with the development of the oxynitride membranes.

The work described in this paper was performed by the Center for Space Microelectronics Technology, Jet Propulsion Laboratory, California Institute of Technology and was jointly sponsored by the Defense Advanced Research Projects Agency, the Strategic Defense Initiative Organization/ Innovative Science and Technology Office, and the National Aeronautics and Space Administration, Office of Aeronautics, Exploration, and Technology.

${ }^{1}$ F. Sibille, Re. Prog. Phys. 49, 1197 (1986).

2 E.H. Putley, in Optical and Infrared Detectors, ed. R.J. Keyes (Springer, Berlin, 1977), pp. 71-98.

${ }^{3}$ T. Limperis, J. Mudar, in The Infrared Handbook, eds. W.L. Wolfe and G.J. Zeiss (Environmental Research Institute of Michigan, Ann Arbor, 1978), Ch. 11.

4 T.G. Blaney, in Infrared and Millimeter Waves Vol. 3, ed. K.J. Button (Academic Press, New York, 1980) pp. 1-75.

5 M.J.E. Golay, Rev. Sci. Inst. 18, 347 (1947).

${ }^{6}$ M.J.E. Golay, Rev. Sci. Inst. 20, 816 (1949).

7 A. Hadni, Essentials of Modern Physics Applied to the Study of the Infrared (Pergamon, Oxford, 1967), pp. 269-283.

8 L.N. Hadley, D.M. Dennison, J. Opt. Sci. Am. 37, 451 (1947).

${ }^{9}$ C. Hilsum, J. Opt. Sci. Am. 44, 188 (1954).

${ }^{10}$ A. Hadni, Essentials of Modern Physics Applied to the Study of the Infrared (Pergamon, Oxford, 1967), pp. 252-7.

11 M.J.S. Smith, L. Bowman, J.D. Meindel, IEEE Trans. Biomed. Eng., BME-33, 163 (1986).

12 S.B. Waltman and W.J. Kaiser, Sensors and Actuators 19, 201 (1989).

13 T.W. Kenny, S.B. Waltman, J.K. Reynolds, and W.J. Kaiser, Appl. Phys. Lett. 58, 100 (1991).

14 G. Binnig, H. Rohrer, Ch. Gerber, and E. Weibel, Phys. Rev. Lett. 49, 57 (1982).

15 G. Binnig, H. Rohrer, Ch. Gerber, and E. Weibel, Phys. Rev. Lett. 50, 120 (1983).

16 W.C. Young, Roark's Formulas for Stress and Strain, 6th Ed. (McGraw-Hill Book Company, New York, 1989).

17 G. Binnig and H. Rohrer, IBM J. Res. Develop. 30, 355 (1986).

18 F.J. Low and A.R Hoffman, Appl. Opt. 2, 649 (1963).

19 R.B. Marcus, T.S. Ravi, T. Gmitter, K. Chin, D. Liu, W.J. Orvis, D.R. Ciarlo, C.E. Hunt and J. Trujillo, Appl. Phys. Lett. 56, 236 (1990). 